\title{
On regular and singular perturbations of acoustic and quantum waveguides
}

\author{
Rustem R. GADYL'SHIN ${ }^{1}$ \\ Bashkir State Pedagogical University, October Revolution St. 3a, \\ 450000, Ufa, Russia \\ Institute of Mathematics, Ufa Science Center of Russian Academy \\ of Sciences, 112 Chernyshevski str., 450077 Ufa, Russia \\ E-mail: gadylshin@bspu.ru, gadylshin@narod.ru
}

\begin{abstract}
We consider regular and singular perturbations of the Dirichlet and Neumann boundary value problems for the Helmholtz equation in $n$ dimensional cylinders. Existence of eigenvalues and their asymptotics are studied.
\end{abstract}

\section{Introduction}

We consider regular and singular perturbations of the Neumann and Dirichlet boundary value problems for $\mathcal{H}_{0}^{(m)}:=-\left(\Delta+\mu_{m}\right)$ in $n$-dimensional cylinder $\Pi=(-\infty, \infty) \times \Omega$, where $\Omega \subset \mathbb{R}^{n-1}$ is a simply connected bounded domain with $C^{\infty}$-boundary for $n \geq 3$ and is an interval $(a, b)$ for $n=2$. Hereinafter, $\mu_{j}$ and $\phi_{j}$ are the eigenvalues and eigenfunctions of $-\Delta^{\prime}:=$ $-\left(\frac{\partial^{2}}{\partial x_{2}^{2}}+\cdots+\frac{\partial^{2}}{\partial x_{n}^{2}}\right)$ in $\Omega$ subject to the same type of the boundary condition on $\partial \Omega$ as in the original unperturbed boundary valued problem for $\mathcal{H}_{0}^{(m)}:=-\left(\Delta+\mu_{m}\right)$ on $\partial \Pi, \mu_{j}<\mu_{j+1}, j=1,2, \ldots$ The functions $\phi_{j}$ are assumed to be normalized in $L^{2}(\Omega)$. The Neumann problem is a mathematical model describing acoustic waveguide while the Dirichlet one corresponds to a quantum waveguide. It is known that unperturbed boundary value problems have no eigenfunctions in $H^{1}(\Pi)$. At the same time such eigenfunctions and eigenvalues (bound states) can emerge under perturbations. We study the

\footnotetext{
${ }^{1}$ The work is supported by grants of RFBR (02-01-00693, 02-01-768) and by the program "Scientific Schools" (1446.2003.1).
} 
questions on existence and absence of such emerging eigenvalues and constructing their asymptotic expansions. Both cases of regular and singular perturbations of these boundary value problems are considered. The regular perturbation treated in the next section is performed by a small localized linear operator of second order. The example of such operator is a small complex potential as well as other perturbations considered in [1] for the Schrödinger operator on the axis. Other examples are small deformations of strips and cylinders which can be reduced to the case we consider by a change of variables [2]-[5]. As a singular perturbation of the Dirichlet and Neumann boundary value problems in $\Pi$ we consider the switching of type of boundary condition at a small segment of the boundary. Such a choice is motivated by a number of articles having appeared recently and containing both rigorous results for quantum waveguides ([3], 6] ) as well as non-rigorous asymptotic results (see [7, 8] and other articles of these authors on singularly perturbed two- and three-dimensional quantum waveguides given in the bibliography of [7, [8]). These formal asymptotics were derived by the method of matching of asymptotic expansions [9] on the basis of scheme employed in [10]-12 for constructing the asymptotics for scattering frequencies of Helmholtz resonator. However, rigorous justification of the asymptotics for these scattering frequencies adduced in [10]-[12] is based on the compactness of obstacle (boundary) and due to this fact it can not be applied to the case of a waveguide. The question on an estimating of the inverse operator for singularly perturbed waveguides (providing a possibility to justify formal asymptotics) is treated in the third section. In two concluding sections we construct the leading terms for asymptotics of the eigenvalues and poles for singularly perturbed quantum and acoustic waveguides.

\section{Regular perturbation}

Hereinafter $H_{l o c}^{j}(\Pi)$ is a set of functions defined on $\Pi$ whose restriction to any bounded domain $D \subset \Pi$ belongs to $H^{j}(D),\|\bullet\|_{G}$ and $\|\bullet\|_{j, G}$ are norms in $L^{2}(G)$ and $H^{j}(G)$, respectively. Next, let $Q=(-R, R) \times \Omega$, where $R>0$ is an arbitrary fixed number, $L^{2}(\Pi ; Q)$ be the subset of functions in $L^{2}(\Pi)$ with supports in $\bar{Q}, \mathcal{L}_{\varepsilon}$ be linear operators mapping $H_{\text {loc }}^{2}(\Pi)$ into $L^{2}(\Pi ; Q)$ such that $\left\|\mathcal{L}_{\varepsilon}[u]\right\|_{Q} \leq C(\mathcal{L})\|u\|_{2, Q}$, where constant $C(\mathcal{L})$ is independent of $\varepsilon, 0<\varepsilon \ll 1$. In this section we study the existence and the asymptotics of the eigenvalues of the Dirichlet and Neumann boundary value problems for $\mathcal{H}_{\varepsilon}^{(m)}:=\mathcal{H}_{0}^{(m)}-\varepsilon \mathcal{L}_{\varepsilon}$ in $\Pi$. For a small complex $k$, we define a linear operator 


$$
\begin{aligned}
& A^{(m)}(k): L^{2}(\Pi ; Q) \rightarrow H_{l o c}^{2}(\Pi) \text { as } \\
& A^{(m)}(k) g:=\left(\sum_{j=1}^{m-1}+\sum_{j=m}^{\infty}\right) \frac{\phi_{j}\left(x^{\prime}\right)}{2 K_{j}^{(m)}(k)} \int_{\Pi} e^{-K_{j}^{(m)}(k)\left|x_{1}-t_{1}\right|} \phi_{j}\left(t^{\prime}\right) g(t) d t,
\end{aligned}
$$

where $x^{\prime}=\left(x_{2}, \ldots, x_{n}\right), K_{j}^{(m)}(k)=\mathrm{i} \sqrt{\mu_{m}-\mu_{j}-k^{2}}$ for $j<m, K_{m}^{(m)}(k)=k$ and $K_{j}^{(m)}(k)=\sqrt{\mu_{j}-\mu_{m}+k^{2}}$ for $j>m$. By analogy with [1] for $f \in$ $L^{2}(\Pi ; Q)$ we seek a solution of

$$
\mathcal{H}_{\varepsilon}^{(m)} u_{\varepsilon}=-k^{2} u_{\varepsilon}+f \quad \text { as } x \in \Pi, \quad u_{\varepsilon}=0 \quad\left(\text { or } \frac{\partial u_{\varepsilon}}{\partial \nu}=0\right) \quad \text { as } x \in \partial \Pi
$$

(where $\nu$ is normal) as

$$
u_{\varepsilon}=A^{(m)}(k) g_{\varepsilon},
$$

where $g_{\varepsilon} \in L^{2}(\Pi ; Q)$. By definition (2.3) is the solution of the equation $\mathcal{H}_{0}^{(m)}(k) u_{\varepsilon}=-k^{2} u_{\varepsilon}+g_{\varepsilon}$ in $\Pi$ and satisfies the boundary condition in (2.2). Substituting (2.3) into (2.2), we get that (2.3) gives a solution for (2.2) if

$$
\left(I-\varepsilon \mathcal{L}_{\varepsilon} A^{(m)}(k)\right) g_{\varepsilon}=f,
$$

where $I$ is identity mapping. If $\mathcal{L}_{\varepsilon}\left[\phi_{m}\right]=0$, due to (2.1), (2.3) and (2.4) it follows that the pole $k_{\varepsilon}^{(m)}$ of (2.3) is equal zero and $g_{\varepsilon} \rightarrow 0$ as $\varepsilon \rightarrow 0$. Thus, there is no small eigenvalue in this case. Assume $\mathcal{L}_{\varepsilon}\left[\phi_{m}\right] \neq 0$,

$$
\begin{gathered}
\langle F\rangle:=\int_{\Pi} F d x, \quad \widetilde{T}_{\varepsilon}^{(m)}(k) g:=\mathcal{L}_{\varepsilon}\left[A^{(m)}(k) g\right]-\frac{\left\langle g \phi_{m}\right\rangle}{2 k} \mathcal{L}_{\varepsilon}\left[\phi_{m}\right], \\
S_{\varepsilon}^{(m)}(k):=\left(I-\varepsilon \widetilde{T}_{\varepsilon}^{(m)}(k)\right)^{-1} .
\end{gathered}
$$

Applying the operator $S_{\varepsilon}^{(m)}(k)$ to both sides of the equation (2.4), we obtain that

$$
\begin{aligned}
& \left(g_{\varepsilon}-\varepsilon \frac{\left\langle g_{\varepsilon} \phi_{m}\right\rangle}{2 k} S_{\varepsilon}^{(m)}(k) \mathcal{L}_{\varepsilon}\left[\phi_{m}\right]\right)=S_{\varepsilon}^{(m)}(k) f \\
& \left\langle g_{\varepsilon} \phi_{m}\right\rangle\left(1-\frac{\varepsilon}{2 k}\left\langle\phi_{m} S_{\varepsilon}^{(m)}(k) \mathcal{L}_{\varepsilon}\left[\phi_{m}\right]\right\rangle\right)=\left\langle\phi_{m} S_{\varepsilon}^{(m)}(k) f\right\rangle .
\end{aligned}
$$

The equality (2.6) allows us to determine $\left\langle g_{\varepsilon} \phi_{m}\right\rangle$. Substituting its value into (2.5), we easily get the formula

$$
g_{\varepsilon}=\varepsilon \frac{2 k\left\langle S_{\varepsilon}^{(m)}(k) f\right\rangle S_{\varepsilon}^{(m)}(k) \mathcal{L}_{\varepsilon}\left[\phi_{m}\right]}{2 k-\varepsilon\left\langle\phi_{m} S_{\varepsilon}^{(m)}(k) \mathcal{L}_{\varepsilon}\left[\phi_{m}\right]\right\rangle}+S_{\varepsilon}^{(m)}(k) f .
$$


Formulas (2.7) and (2.3) imply, that, if $k_{\varepsilon}^{(m)}$ is a solution of the equation

$$
2 k-\varepsilon\left\langle\phi_{m} S_{\varepsilon}^{(m)}(k) \mathcal{L}_{\varepsilon}\left[\phi_{m}\right]\right\rangle=0,
$$

then the residue of (2.3) at $k_{\varepsilon}^{(m)}$ :

$$
\psi_{\varepsilon}^{(m)}=A^{(m)}\left(k_{\varepsilon}^{(m)}\right) S_{\varepsilon}^{(m)}\left(k_{\varepsilon}^{(m)}\right) \mathcal{L}_{\varepsilon}\left[\phi_{m}\right]
$$

is the solution of the equation $\mathcal{H}_{\varepsilon}^{(m)} \psi_{\varepsilon}^{(m)}=\lambda_{\varepsilon}^{(m)} \psi_{\varepsilon}^{(m)}$ in $\Pi$ (with corresponding homogeneous Dirichlet or Neumann boundary conditions), where $\lambda_{\varepsilon}^{(m)}=$ $-\left(k_{\varepsilon}^{(m)}\right)^{2}$. The formulas (2.1), (2.9) yield if $\operatorname{Re} k_{\varepsilon}^{(1)}>0$, then $\psi_{\varepsilon}^{(1)} \in L^{2}(\Pi)$ and, hence, $\lambda_{\varepsilon}^{(1)}$ is the eigenvalue which due to (2.8) has the asymptotics

$$
\lambda_{\varepsilon}^{(m)}=-\varepsilon^{2} \frac{1}{4}\left\langle\phi_{m} \mathcal{L}_{\varepsilon}\left[\phi_{m}\right]\right\rangle^{2}+O\left(\varepsilon^{3}\right)
$$

with $m=1$ (and the function (2.9) is the associated eigenfunction). For $m \geq$ 2, the formulas (2.1), (2.8), (2.9) imply, that if $\operatorname{Re} k_{\varepsilon}^{(m)}>0$ and $\operatorname{Im} k_{\varepsilon}^{(m)}>0$, then $\psi_{\varepsilon}^{(m)} \in L^{2}(\Pi)$, too, and, hence, $\lambda_{\varepsilon}^{(m)}$ is the eigenvalue of the perturbed problem with asymptotics (2.10). In particular, the equation (2.8) allows us to maintain that in the case $\left\langle\phi_{1} \mathcal{L}_{\varepsilon}\left[\phi_{1}\right]\right\rangle \geq \delta>0$ there exists small eigenvalue.

\section{Singular perturbations. Convergence of poles and representation of solutions near poles}

Assume for simplicity in describing the of perturbations that the domain $\Omega$ coincides with half-space $x_{n}>0$ in some neighborhood of the origin (in variables $\left.x^{\prime}\right), \omega$ is a $(n-1)$-dimensional bounded domain in the hyperplane $x_{n}=0$ having smooth boundary, $\omega_{\varepsilon}=\left\{x: x \varepsilon^{-1} \in \omega\right\}, \Gamma_{\varepsilon}=\partial \Pi \backslash \overline{\omega_{\varepsilon}}$. For a given $f \in L^{2}(\Pi ; Q)$, we consider two singularly perturbed boundary value problems

$$
\begin{aligned}
& \mathcal{H}_{0}^{(m)} u_{\varepsilon}=-k^{2} u_{\varepsilon}+f, \quad x \in \Pi, \\
& u_{\varepsilon}=0, \quad x \in \Gamma_{\varepsilon}\left(\text { or } x \in \omega_{\varepsilon}\right), \quad \frac{\partial u_{\varepsilon}}{\partial \nu}=0, \quad x \in \omega_{\varepsilon}\left(\text { or } x \in \Gamma_{\varepsilon}\right) .
\end{aligned}
$$

Let $\Gamma_{0}^{R}=\partial \Pi \cap \partial Q, \Omega^{R}=\partial Q \backslash \overline{\Gamma_{0}^{R}}, \Gamma_{\varepsilon}^{R}=\Gamma^{R} \backslash \overline{\omega_{\varepsilon}}$. For each $V \in H^{2}(Q)$, we denote by $\sigma_{\varepsilon}: H^{2}(Q) \rightarrow H^{1}(Q)$ the inverse operator for the following boundary value problems

$$
\begin{aligned}
\Delta W_{\varepsilon} & =\Delta V, \quad x \in Q, \quad W_{\varepsilon}=V, \quad x \in \Omega^{R}, \\
W_{\varepsilon} & =0, \quad x \in \Gamma_{\varepsilon}^{R}\left(\text { or } x \in \omega_{\varepsilon}\right), \quad \frac{\partial W_{\varepsilon}}{\partial \nu}=0, \quad x \in \omega_{\varepsilon}\left(\text { or } x \in \Gamma_{\varepsilon}^{R}\right) .
\end{aligned}
$$


Let $\chi^{ \pm}\left(x_{1}\right)$ be an infinitely differentiable mollifier function equalling to one for $\pm x_{1} \leq R / 2$ and vanishing for $\pm x_{1} \geq R, \Pi_{ \pm}=\left\{x: x \in \Pi, \pm x_{1}>0\right\}$, $p_{ \pm}$be the restriction operator from $\Pi$ to $\Pi_{ \pm}, p_{ \pm}^{\bar{Q}}$ be the restriction operator from $\Pi_{ \pm}$to $\Pi_{ \pm} \cap Q$,

$$
\begin{aligned}
A_{ \pm}^{(m)}(k) g^{ \pm}:= & \sum_{j=1}^{\infty} \frac{\phi_{j}\left(x^{\prime}\right)}{2 K_{j}^{(m)}(k)} \int_{\Pi_{ \pm}}\left(e^{-K_{j}^{(m)}(k)\left|x_{1}-t_{1}\right|}\right. \\
& \left.-e^{-K_{j}^{(m)}(k)\left|x_{1}+t_{1}\right|}\right) \phi_{j}\left(t^{\prime}\right) g^{ \pm}(t) d t, \quad x \in \Pi_{ \pm}, \\
\mathcal{A}_{\varepsilon}^{(m)}(k) g:= & \left(1-\chi^{+}\right) A_{+}^{(m)}(k) p_{+} g+\left(1-\chi^{-}\right) A_{-}^{(m)}(k) p_{-} g \\
& +\chi^{+} \chi^{-} \sigma_{\varepsilon}\left(p_{+}^{Q} A_{+}^{(m)}(k) p_{+} g+p_{-}^{Q} A_{-}^{(m)}(k) p_{-} g\right), \quad g \in L^{2}(\Pi ; Q) .
\end{aligned}
$$

We construct the solution of (3.1) in the form

$$
u_{\varepsilon}=\mathcal{A}_{\varepsilon}^{(m)}(k) g_{\varepsilon},
$$

where $g_{\varepsilon}$ is a some function belonging to $L^{2}(\Pi ; Q)$. Substituting (3.2) into (3.1), by analogy with [13] we deduce that this function is a solution of (3.1) in the case

$$
g_{\varepsilon}=\left(I+T_{\varepsilon}^{(m)}(k)\right)^{-1} f,
$$

where, for any fixed $\varepsilon, T_{\varepsilon}^{(m)}(k)$ is a holomorphic operator-valued function and, for any fixed $k, T_{\varepsilon}^{(m)}(k)$ is a compact operator in $L^{2}(\Pi ; Q)$. Analyze of this family with respect to $\varepsilon$ (which is similar to [14] and based on [13]) and the representations (3.2), (3.3) imply that there exists one pole $k_{\varepsilon}^{(m)} \rightarrow 0$ of the solution of (3.1) and for small $k$, this solution meet the representation

$$
\begin{gathered}
u_{\varepsilon}(x, k)=\frac{\psi_{\varepsilon}^{(m)}(x)}{2\left(k-k_{\varepsilon}^{(m)}\right)} \int_{\Pi} \psi_{\varepsilon}^{(m)}(y) f(y) d y+\widetilde{u}_{\varepsilon}(x, k), \\
\left\|\widetilde{u}_{\varepsilon}\right\|_{1, D} \leq C(D, Q)\|f\|_{\Pi}
\end{gathered}
$$

for any bounded domain $D \subset \Pi$. The residue $\psi_{\varepsilon}^{(m)}$ at this pole is a solution to the equation $\mathcal{H}_{0}^{(m)} \psi_{\varepsilon}^{(m)}=\lambda_{\varepsilon}^{(m)} \psi_{\varepsilon}^{(m)}$ in $\Pi$, where $\lambda_{\varepsilon}^{(m)}=-\left(k_{\varepsilon}^{(m)}\right)^{2}$, satisfies the boundary conditions from (3.1) and for any fixed $x_{1}$ converges to $\phi_{m}$ (up to a multiplicative constant) as $\varepsilon \rightarrow 0$. This convergence, the representation (3.2) and the definition of $\mathcal{A}_{\varepsilon}^{(m)}(k)$ imply that

$$
\begin{gathered}
\psi_{\varepsilon}^{(m)}(x)=\sum_{j=1}^{m-1} a_{j}^{\varepsilon} \phi_{j}\left(x^{\prime}\right) e^{-\left|x_{1}\right| \operatorname{Re} K_{j}^{(m)}\left(k_{\varepsilon}^{m}\right)}+a_{m}^{\varepsilon} \phi_{m}\left(x^{\prime}\right) e^{-\left|x_{1}\right| \operatorname{Re} k_{\varepsilon}^{(m)}}+o\left(e^{-\left|x_{1}\right| \delta}\right) \\
\text { as }\left|x_{1}\right| \rightarrow \infty
\end{gathered}
$$


where $a_{m}^{\varepsilon}=1+o(1)$ as $\varepsilon \rightarrow 0$ and $\delta>0$ some fixed number. In partially, this asymptotics implies that

$$
\begin{aligned}
& \text { there exists eigenvalue } \lambda_{\varepsilon}^{(1)} \text { provided } \operatorname{Re} k_{\varepsilon}^{(1)}>0, \\
& \text { if } m \geq 2, \operatorname{Re} k_{\varepsilon}^{(m)}>0 \text { but } \operatorname{Im} k_{\varepsilon}^{(m)}<0 \text { and } a_{1}^{\varepsilon} \neq 0, \\
& \text { then there is no an eigenvalue, } \\
& \text { there is no an eigenvalue if } \operatorname{Re} k_{\varepsilon}^{(m)} \leq 0 .
\end{aligned}
$$

Thus, in fact we need to construct and to justify asymptotics of the pole $k_{\varepsilon}^{(m)}$ (and, an additional, asymptotics of the residue $\psi_{\varepsilon}^{(m)}$ in the case (3.7)) which generates the eigenvalue or doesn't. As above mentioned in the case of regular perturbation the asymptotics for pole can obtained by simple calculations in (2.8), while dealing with singular perturbation, we have no such equation. On the other hand, the representation (3.5) allows to justify the method of matching asymptotic expansions in constructing the asymptotics for the poles $k_{\varepsilon}^{(m)}$ and for the residue $\psi_{\varepsilon}^{(m)}$.

As it has been mentioned above, the formal construction of complete asymptotics of poles for the boundary valued problems (3.1) and for Helmholtz resonator [10]-12] is similar. That's why in the next two section we will construct first perturbed terms of poles only.

\section{Singular perturbation of quantum waveg- uide. Asymptotics of poles and eigenvalues}

Let $S_{n}$ be the unit sphere in $\mathbb{R}^{n}, G_{m}^{(\mathcal{D})}(x, y, k)$ be the Green function of the unperturbed Dirichlet boundary value problem in $\Pi, \Phi_{m}=\left.\frac{\partial}{\partial x_{n}} \phi_{m}\left(x^{\prime}\right)\right|_{x^{\prime}=0} \neq$ $0, \Psi_{m}^{(\mathcal{D})}(x, k)=-\left.2 k \Phi_{m}^{-1} \frac{\partial}{\partial y_{n}} G_{m}^{(\mathcal{D})}(x, y, k)\right|_{y=0}$. By definition

$$
\begin{aligned}
& \Psi_{m}^{(\mathcal{D})}(x, k) \rightarrow \phi_{m}\left(x^{\prime}\right), \quad k \rightarrow 0 \quad \text { for any fixed } x \neq 0, \\
& \Psi_{m}^{(\mathcal{D})}(x, k)=\Phi_{m} x_{n}+\frac{4 k}{\Phi_{m}\left|S_{n}\right|} \frac{x_{n}}{r^{n}}+O\left(k r^{-n+2}\right), \quad r=|x| \rightarrow 0, k \rightarrow 0 .
\end{aligned}
$$

Taking into account (4.1), outside small neighborhood of $\omega_{\varepsilon}$ we construct the residue $\psi_{\varepsilon}^{(m)}$ in the form $\psi_{\varepsilon}^{(m)}(x) \sim \Psi_{m}^{(\mathcal{D})}\left(x, k_{\varepsilon}^{(m)}\right)$. Near $\omega_{\varepsilon}$ we construct asymptotics by using the method of matching asymptotic expansions [9]- 12 ] in the variables $\xi=\varepsilon^{-1} x$. The structure of the expansions of $\psi_{\varepsilon}^{(m)}$ in this zone and of the pole $k_{\varepsilon}^{(m)}$ are inspired by the following consideration. When $x=\varepsilon \xi$ and $k=k_{\varepsilon}^{(m)}$, both terms in right hand side of (4.2) must have 
the same order with respect to $\varepsilon$. This degree determines the first term in the interior layer for $\psi_{\varepsilon}^{(m)}$, while the right hand side of (4.2) (rewritten in variables $\xi$ and for $k=k_{\varepsilon}^{(m)}$ ) determines the asymptotics of this term as $\rho=|\xi| \rightarrow \infty$. Due to these reasons we construct asymptotics as

$$
\begin{aligned}
k_{\varepsilon}^{(m)} & =\varepsilon^{n} \tau_{n}^{(m)}+\ldots, \quad \psi_{\varepsilon}^{(m)}(x)=\varepsilon v_{1}^{(m)}(\xi)+\ldots, \\
v_{1}^{(m)}(\xi) & =\Phi_{m} \xi_{n}+4 \tau_{n}^{(m)}\left(\Phi_{m}\left|S_{n}\right|\right)^{-1} \xi_{n} \rho^{-n}+o\left(\rho^{-n+1}\right), \quad \rho \rightarrow \infty .
\end{aligned}
$$

Substituting (4.3) in (3.1) (with $f=0$ and $k=k_{\varepsilon}^{(m)}$ ), we obtain the boundary value problem for $v_{1}^{(m)}$ :

$$
\Delta_{\xi} v_{1}^{(m)}=0, \quad \xi_{n}>0, \quad v_{1}^{(m)}=0, \quad \xi \in \Gamma, \quad \frac{\partial v_{1}^{(m)}}{\partial \xi_{n}}=0, \quad \xi \in \omega,
$$

where $\Gamma=\left\{\xi: \xi_{n}=0, \xi \notin \omega\right\}$. It is known, there exists the solution $X_{n}$ of (4.5) with asymptotics $X_{n}(\xi)=\xi_{n}+c_{n}(\omega) \xi_{n} \rho^{-n}+o\left(\rho^{-n+1}\right)$ as $\rho \rightarrow \infty$, where $c_{n}(\omega)>0$. Thus it follows from (4.4) that

$$
v_{1}^{(m)}(\xi)=\Phi_{m} X_{n}(\xi), \quad \tau_{n}^{(m)}=4^{-1} c_{n}(\omega)\left|S_{n}\right| \Phi_{m}^{2}>0 .
$$

By (4.3), (4.6) we have $\operatorname{Re} k_{\varepsilon}^{(m)}>0$ and, hence (see (3.6)), there exists eigenvalue

$$
\lambda_{\varepsilon}^{(1)}=-\varepsilon^{2 n}\left(\frac{c_{n}(\omega)\left|S_{n}\right| \Phi_{1}^{2}}{4}\right)^{2}+o\left(\varepsilon^{2 n}\right) .
$$

For $m \geq 2$, constructing next terms for expansions $k_{\varepsilon}^{(m)}$ and $\psi_{\varepsilon}^{(m)}$ (similar [10]-12]) one can obtain that

$$
\begin{aligned}
\operatorname{Im} k_{\varepsilon}^{(m)} & =-\varepsilon^{2 n}\left(\frac{c_{n}(\omega)\left|S_{n}\right| \Phi_{m}}{4}\right)^{2} \sum_{j=1}^{m-1} \frac{\Phi_{j}^{2}}{\sqrt{\mu_{m}-\mu_{j}}}+o\left(\varepsilon^{2 n}\right)<0, \\
a_{1}^{\varepsilon} & \sim \frac{k_{\varepsilon}^{(m)} \Phi_{1}}{K_{1}^{(m)}\left(k_{\varepsilon}^{(m)}\right) \Phi_{m}} \neq 0 .
\end{aligned}
$$

where $\Phi_{j}=\left.\frac{\partial}{\partial x_{n}} \phi_{j}\left(x^{\prime}\right)\right|_{x^{\prime}=0}$. Therefore, the pole $k_{\varepsilon}^{(m)}$ meets the asymptotics (4.3), (4.6), but (see (3.7)) does not generate an eigenvalue of the considered singular perturbation of the Dirichlet boundary value problem.

\section{Singular perturbation of acoustic waveg- uide. Asymptotics of poles}

Let $G_{m}^{(\mathcal{N})}(x, y, k)$ be the Green function of the unperturbed Neumann boundary value problem, $\phi_{m}(0) \neq 0, \Psi_{m}^{(\mathcal{N})}(x, k)=-2 k \phi_{m}^{-1}(0) G_{m}^{(\mathcal{N})}(x, 0, k), \alpha_{n}(r)=$ 
$r^{-n+2}$ for $n \geq 3$ and $\alpha_{2}(r)=-\ln r$. By definition

$$
\begin{gathered}
\Psi_{m}^{(\mathcal{N})}(x, k) \rightarrow \phi_{m}\left(x^{\prime}\right), \quad k \rightarrow 0 \quad \text { for any fixed } x \neq 0, \\
\Psi_{m}^{(\mathcal{N})}(x, k)=\phi_{m}(0)+4 k\left(\phi_{m}(0)\left|S_{n}\right|\right)^{-1} \alpha_{n}(r)+O\left(k r^{-n+3-\delta_{n}^{2}}\right), \\
r \rightarrow 0, k \rightarrow 0,
\end{gathered}
$$

where $\delta_{J}^{s}$ is the Cronecker delta. Taking into account (5.1) and following the method of matching asymptotic expansions similar the previous section we obtain that

$$
\begin{aligned}
& k_{\varepsilon}^{(m)}=\varepsilon^{n-2} \tau_{n-2}^{(m)}+\ldots, \quad n \geq 3, \quad k_{\varepsilon}^{(m)}=-\ln ^{-1} \varepsilon \tau_{0}^{(m)}+\ldots, \quad n=2, \\
& \tau_{n-2}^{(m)}=-\frac{C_{n}(\omega)\left|S_{n}\right| \phi_{m}^{2}(0)}{4}<0, \quad n \geq 3, \quad \tau_{0}^{(m)}=-\frac{\pi \phi_{m}^{2}(0)}{2}<0, \quad n=2,
\end{aligned}
$$

where $C_{n}(\omega)>0$ is the capacity of the disk $\omega$. Thus, Re $k_{\varepsilon}^{(m)}<0$. Therefore, the pole $k_{\varepsilon}^{(m)}$ meets the asymptotics (5.2), but (see (3.8)) it does not generate an eigenvalue of the considered singular perturbation of the Neumann boundary value problem.

The author thanks E. Sanchez-Palencia and D. I. Borisov for discussion of the work and useful remarks. The work is supported by grants of RFBR (02-01-00693, 02-01-00768) and by the program "Scientific Schools" (1446.2003.1).

\section{References}

[1] Gadyl'shin R.R., On local perturbations of Shrödinger operator in axis, Teor. Mat. Fiz. 132 (1) (2002) 97-104. English translation: Theor. Math. Phys. 132 (1) (2002) 976-982.

[2] Duclos P., Exner P., Curvature-induced bound states in quantum waveguides in two and three dimensions, Rev. Math. Phys. 7 (1995) 73-102.

[3] Bulla W., Gesztesy F., Renger W., Simon B., Weakly coupled bound states in quantum waveguides, Proc. Amer. Math. Soc. 127 (1997) 14871495.

[4] Exner P., Vugalter S.A, Bound states in a locally deformed waveguide: the critical case, Lett. Math. Phys. 39 (1997) 59-68. 
[5] Borisov D., Exner P., Gadyl'shin R., Krejcirik D., Bound states in weakly deformed strips and layers, Ann. H. Poincaré 2 (3) (2001) 553-572.

[6] Exner P., Vugalter S.A., Asymptotic estimates for bound states in quantum waveguides coupled laterally through a narrow window, Ann. Inst. H. Poincaré: Phys. théor. 65 (1996) 109-123.

[7] Popov I.Yu. Asymptotics of bounded states for laterally coupled threedimensional waveguides, Rep. Math. Phys. 48 (2001) 277-288.

[8] Popov I.Yu., Frolov S.V., Three laterally coupled quantum waveguiedes: breaking of symmetry and resonance asymptotics, J. Phys. A: Math. Gen. 36 (2003) 1655-1670.

[9] Il'in A.M., Matching of Asymptotic Expansions of Solutions of Boundary Value Problems, American Mathematical Society, Providence, RI, 1992.

[10] Gadyl'shin R.R., Surface potentials and the method of matching asymptotic expansions in the problem of the Helmholtz resonator, Algebra Anal. 4 (2) (1992) 88-115. English translation: St. Petersbg. Math. J. 4 (1) (1993) 273-296.

[11] Gadyl'shin R.R., On acoustic Helmholtz resonator and on its electromagnetic analog, J. Math. Phys. 35 (7) (1994) 3464-3481.

[12] Gadyl'shin R.R., Existence and asymptotics of poles with small imaginary part for the Helmholtz resonator, Usp. Mat. Nauk 52 (1) (1997) 3-76. English translation: Russ. Math. Surv. 52 (1) (1997) 1-72.

[13] Sanchez-Palencia E., Non-Homogeneous Media and Vibration Theory, Springer-Verlag, New-York, 1980.

[14] Gadyl'shin R.R., On analogs of Helmholtz resonator in averaging theory, Mat. Sbor. 193 (11) (2002) 43-70. English translation: Sb. Math. 193 (11) (2002) 1611-1638. 